\title{
EXPERIMENTAL RESULTS ON THE VIBRATORY ENERGY EXCHANGES BETWEEN A LINEAR SYSTEM AND A CHAIN OF NONLINEAR OSCILLATORS
}

A PREPRINT: HTTPS://DOI.ORG/10.1016/J.JSV.2018.09.004

\author{
C.-H. Lamarque \\ Univ Lyon, ENTPE, LTDS UMR CNRS 5513 \\ Rue Maurice Audin, F-69518 Vaulx-en-Velin Cedex, France \\ lamarque@entpe.fr \\ A. Ture Savadkoohi* \\ Univ Lyon, ENTPE, LTDS UMR CNRS 5513 \\ Rue Maurice Audin F-69518 Vaulx-en-Velin Cedex, France \\ alireza.turesavadkoohi@entpe.fr \\ S. Charlemagne \\ Univ Lyon, ENTPE, LTDS UMR CNRS 5513 \\ Rue Maurice Audin F-69518 Vaulx-en-Velin Cedex, France \\ simon. charlemagne@developpement-durable.gouv.fr
}

\begin{abstract}
Experimental results on a nonlinear chain coupled to a main system are presented. The chain is composed of eight moving masses, each one possesses local nonlinear restoring forcing function and global linear springs for coupling to other masses. The main system is coupled to the first mass of the chain via a linear spring. The main system is under external sinusoidal excitation with sweeping frequency around its targeted mode. Experimental results show that according to the amplitude of the excitation, the system can reach periodic or modulated regimes. The goal of using the nonlinear chain is to examine experimentally the possibility of localization of the vibratory energy of the main system into the chain for the aim of passive control.
\end{abstract}

Keywords experimental study $\cdot$ passive control $\cdot$ localization $\cdot$ nonlinear chain $\cdot$ strongly modulated response . periodic regime

\section{Introduction}

The use of additional nonlinear devices has shown its efficiency in terms of passive control of primary structures (1). Indeed, essential nonlinearity enables secondary systems such as Nonlinear Energy Sink (NES) (2, 3, 4) to enter into resonance with any frequency, allowing a broadband control. Studying nonlinear interactions between nonlinear oscillators in general and multiple NES systems in detail has quite extensive literature. Some works have considered three degree-of-freedom (dof) vibration absorbers, showing their capacity to perform a multi-modal control of impulseloaded systems $(5 ; 6$; 7). Some researches concentrated on resonance energy exchanges between particles with the concept of limiting phase trajectories (8). A detailed overview in the area of ordered granular media such as dimer and weakly coupled chains and locally resonant acoustic metamaterials can be found in (9).

In previous works, we detailed an analytical methodology for studying nonlinear chains of light oscillators coupled to a primary structure under harmonic excitation, in which the chain could be examined via a continuous approximation

\footnotetext{
${ }^{*}$ Corresponding author
} 
$(10 ; 11 ; 12)$. The current paper presents experimental results on a system composed of a linear master structure (MS) couple to a nonlinear chain of oscillators. Each mass of the chain possesses nonlinear local and linear global restoring forcing terms. For practical reasons, the experimental system is slightly different from the theoretical case studied one by Lamarque et al.(11) and Charlemagne et al.(12). The paper is organized as follows: The general mechanical model of the system under consideration and its governing equations are provided in Sect. 2. The realization and characterization of the overall system are explained in Sect. 3 In detail: the experimental characterization of the MS is clarified in Sect. 3.1 while the fabrication process of the chain and its local and global springs are provided in Sect. 3.2. The characterizations of the coupled systems are illustrated in Sect. 3.3 Experimental results on the chain alone and also the system of coupled oscillators, i.e. linear system and the chain are provided in Sect. 4. Finally, the paper is concluded in Sect. 5

\section{The mechanical model of the system under consideration}

The general theoretical representation of the considered system is shown in Fig. 1. It consists of a linear oscillator, representing the MS with the mass $M$, stiffness $K$ and damping $C$, subjected to an external solicitation $F(t)=$ $A_{M S} \sin (\omega t)$. A chain of nonlinear oscillators of equal masses for each particle as $m=\epsilon M(0<\epsilon \ll 1)$ is coupled to the MS. Each mass of the chain possesses a local restoring forcing term, $\tilde{G}(z)$, which is sum of linear and cubic functions, reads as $\tilde{G}(z)=\tilde{B}_{l} z+\tilde{V} z^{3}$. The global coupling between each mass is carried out via a linear spring with the resorting forcing function and viscous damping as $\tilde{B}$ and $\Gamma$, respectively (12). Governing system equations at the

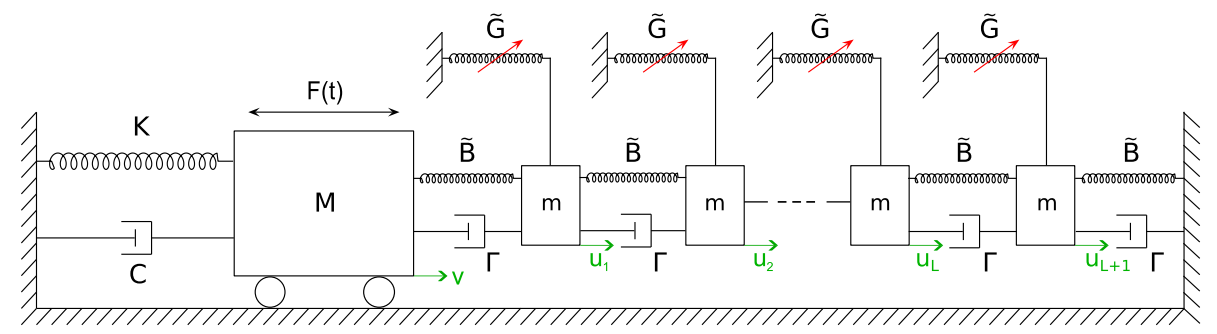

Figure 1: $(L+2)$ dof system consisting of a forced linear main structure coupled to $(L+1)$ light nonlinear oscillators $(m=\epsilon M, 0<\epsilon \ll 1)$.

time domain $t$ are summarized as:

$$
\left\{\begin{array}{l}
M \ddot{v}+C \dot{v}+K v+\Gamma\left(\dot{v}-\dot{u}_{1}\right)+\tilde{B}\left(v-u_{1}\right)=A_{M S} \sin (\omega t) \\
m \ddot{u}_{1}+\Gamma\left(-\dot{v}+2 \dot{u}_{1}-\dot{u}_{2}\right)+\tilde{B}\left(-v+2 u_{1}-u_{2}\right)+\tilde{B}_{l} u_{1}+\tilde{V} u_{1}^{3}=0 \\
\vdots \\
m \ddot{u}_{j}+\Gamma\left(-\dot{u}_{j-1}+2 \dot{u}_{j}-\dot{u}_{j+1}\right)+\tilde{B}\left(-u_{j-1}+2 u_{j}-u_{j+1}\right)+\tilde{B}_{l} u_{j}+\tilde{V} u_{j}^{3}=0 \quad, \quad j=2, \ldots, L \\
\vdots \\
m \ddot{u}_{L+1}+\Gamma\left(2 \dot{u}_{L+1}-\dot{u}_{L}\right)+\tilde{B}\left(2 u_{L+1}-u_{L}\right)+\tilde{B}_{l} u_{L+1}+\tilde{V} u_{L+1}^{3}=0
\end{array}\right.
$$

where $v$ and $u_{j}, j \in[|0, L+1|]$, stand for the displacements of the MS and the oscillators of the chain, respectively. Let us set the linear frequency of the main system as $\omega_{0}=\sqrt{\frac{K}{M}}$, and define a new time domain as $T=\omega_{0} t$. We rescale system variables as: $\tilde{v}=\frac{v}{x_{0}}$ and $\tilde{u}_{j}=\frac{u_{j}}{x_{0}}$. Governing system equations read:

$$
\left\{\begin{array}{l}
\ddot{\tilde{v}}+\epsilon c \dot{\tilde{v}}+\tilde{v}+\epsilon \gamma\left(\dot{\tilde{v}}-\dot{\tilde{u}}_{1}\right)+\epsilon B\left(\tilde{v}-\tilde{u}_{1}\right)=\epsilon f \sin (\Omega T) \\
\ddot{\tilde{u}}_{1}+\gamma\left(-\dot{\tilde{v}}+2 \dot{\tilde{u}}_{1}-\dot{\tilde{u}}_{2}\right)+B\left(-\tilde{v}+2 \tilde{u}_{1}-\tilde{u}_{2}\right)+B_{l} \tilde{u}_{1}+D \tilde{u}_{1}^{3}=0 \\
\vdots \\
\ddot{\tilde{u}}_{j}+\gamma\left(-\dot{\tilde{u}}_{j-1}+2 \dot{\tilde{u}}_{j}-\dot{\tilde{u}}_{j+1}\right)+B\left(-\tilde{u}_{j-1}+2 \tilde{u}_{j}-\tilde{u}_{j+1}\right)+B_{l} \tilde{u}_{j}+D \tilde{u}_{j}^{3}=0, \quad j=2, \ldots, L \\
\vdots \\
\ddot{\tilde{u}}_{L+1}+\gamma\left(2 \dot{\tilde{u}}_{L+1}\right)+B\left(2 \tilde{u}_{L+1}-\tilde{u}_{L}\right)+B_{l} \tilde{u}_{L+1}+B_{l} \tilde{u}_{L+1}+D \tilde{u}_{L+1}^{3}=0
\end{array}\right.
$$


with $\frac{\tilde{B}}{K}=\epsilon B, \frac{\tilde{B}_{l}}{K}=\epsilon B_{l}, \frac{\tilde{V} x_{0}^{2}}{K}=\epsilon D, \frac{C}{\sqrt{K M}}=\epsilon c, \frac{\Gamma}{\sqrt{K M}}=\epsilon \gamma, \frac{A_{M S}}{K x_{0}}=\epsilon f$ and $\Omega=\frac{\omega}{\omega_{0}}$.

A summary of different steps for analytical treatments of the system 2 is provided in Appendix. Globally speaking, the methodology for designing such nonlinear systems and also studying energy exchanges between oscillators rely on detection of the slow invariant manifold (SIM) (13) of the system completed by tracing equilibrium and singular points (18). The geometry of the SIM which can be tuned by parameters of nonlinear oscillators (such as coefficient of the nonlinear terms of the restoring forcing function) can be chosen in such a manner that the system, for given initial conditions and/or under spacial external excitation characteristics, presents extreme nonlinear interactions between oscillators followed by bifurcations and leading to different regimes (17). Bifurcations of the system are due the existence of unstable zone(s) (one or several unstable zones (10)) of the SIM. These bifurcations normally correspond to large and abrupt variations of energy of nonlinear oscillators and very small variations of the energy of main linear system. If the goal of using nonlinear oscillators is passive control of the main system, then the equilibrium and singular points should correspond to regimes with low energy interval(s) for the main system. The current paper focuses on test results on a experimental device that has been built in agreement with this model to be investigated. Detailed analytical developments and predictions are out of the scope of the current work.

\section{Experimental test set-up}

In this section the realization procedure of the presented mechanical model in Fig. 1 is described. It covers the realization and characteristics identification of the MS, the chain and creations of its local and global springs.

\subsection{Description of the MS and its characterization}

The schematic of the MS is shown in Fig. 2. It consists of a parallelepipedal block of steel resting on four identical columns made up of steel. In fact this model is endowed for representing a targeted mode of a general model of a structure to be controlled. As it is shown in Fig. 2, the main system is clamped at its basis on a plate in Plexiglas. The plate is fixed on the shaking table of the ENTPE that permits to excite the structure at its base in the $X$ direction (see Fig. 2). Dimensions of the components of the scaled model in $X, Y$ and $Z$ directions are given in Table 1 Dimensions of the parallelepiped are provided without taking into account the $2 \mathrm{~mm}$ slot spaces needed towards the joint with columns. The height $Z$ of columns corresponds to the free length, i.e. from the basis of the parallelepiped

\begin{tabular}{|c||c|c|c|}
\hline Dimensions $(\mathrm{mm})$ & $\mathrm{X}$ & $\mathrm{Y}$ & $\mathrm{Z}$ \\
\hline Parallelepiped & 100 & 100 & 40 \\
\hline Column & 2 & 10 & 85 \\
\hline
\end{tabular}

Table 1: Dimensions of the components of the scale model.

to the plate in Plexiglas in which each pin is clamped on $10 \mathrm{~mm}$. From these dimensions and because of the direction of the excitation of the shaking table, we can assume that :

- The parallelepiped is undeformable.

- Displacements occur mainly in the X direction.

- Eigenfrequencies of the columns (seen as clamped-clamped beams) are much greater than the eigenfrequencies examined for the coupled system.

The scaled model of the main system can be represented by a linear single dof oscillator with mass $M$ and spring stiffness $K$. Its displacements are weak enough to carry on a linear elastic behaviour. Dissipation is taken into account via a viscous damping with coefficient $C$ (unknown, to be estimated). The mass $M$ is evaluated as the sum of the mass of the parallelepiped and half of the masses of the four columns, resulting as $M=3.18 \mathrm{~kg}$. To evaluated $K$, we use the idealized two-dimensional mechanical model of the main system which is depicted in Fig. 3 . The equivalent lateral stiffness of the frame, i.e. $K$ assuming that the rigidity of the floor $(E I)$ is very large compared to the ones of connecting elements, or columns (EI) with the length $L_{b}$ is obtained as (19):

$$
K=4 K_{b}=4 \frac{12 E I}{L_{b}^{3}}=1.094 \times 10^{5} \mathrm{~N} \cdot \mathrm{m}^{-1}
$$




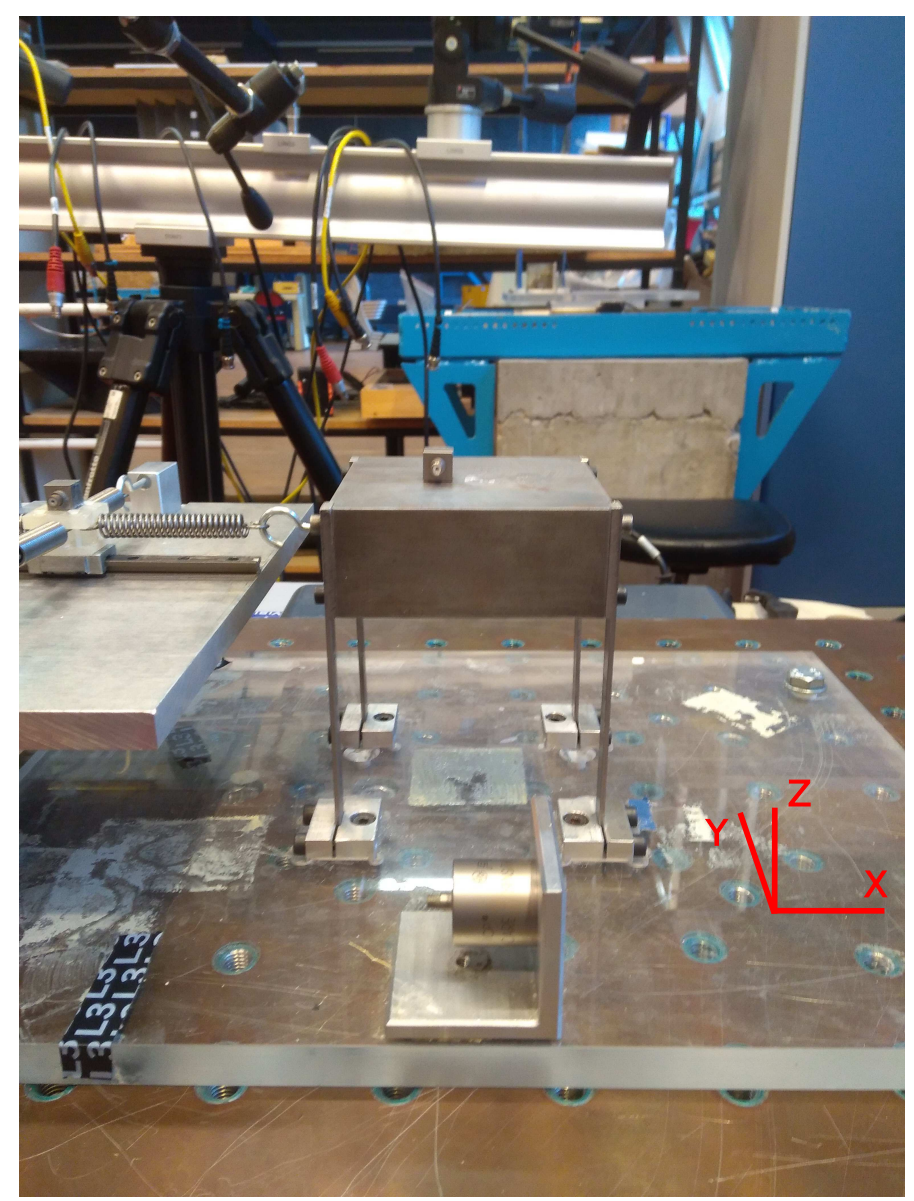

Figure 2: The picture of the MS used for the experiments. The main mass is mounted on four columns and is linked to the first small mass of the chain on the left.

where $I$ is the second moment of inertia versus $Y, L_{b}$ its height versus $\mathrm{Z}$ and $E$ is the Young's modulus of the steel. The eigenfrequency of the main system is given by:

$$
\nu_{0}=\frac{\omega_{0}}{2 \pi}=\frac{1}{2 \pi} \sqrt{\frac{K}{M}}=29.52 \mathrm{~Hz}
$$

An accelerometer is placed on the Plexiglas plate to record input acceleration while another accelerometer located on top of the parallelepiped records the output acceleration, see Fig. 2. To identify the frequency of the main system, the shaking table is excited by a white noise with constant amplitude and a sweeping frequencies between $5 \mathrm{~Hz}$ to $500 \mathrm{~Hz}$. Obtained power spectral density is presented in Fig. 4 showing an identified frequency of the system at $\nu_{0}=27.5 \mathrm{~Hz}$, which is slightly different from the theoretically evaluated frequency in Eq. 4

\subsection{Description of the nonlinear chain and its realization}

For realization of the mechanical model of the nonlinear local potential of the chain which is represented in Fig. 1 the following technique is used (14, 15; 16): two linear springs with rigidity as $k$ and initial length as $L_{i}$, pre-stressed by the force $P$ are coupled to a mass which moves on the orthogonal direction of springs as illustrated in Fig. 5. Due to the applied traversal forcing term $F$ on the mass, it experiences the displacement $u$ which makes the length of spring becomes $L_{f}$. Under the hypothesis of small deformations, one can write:

$$
F=\frac{2 u}{L_{f}}\left(P+k\left(L_{f}-L_{i}\right)\right)
$$

with

$$
L_{f}=\sqrt{u^{2}+L_{i}^{2}}
$$




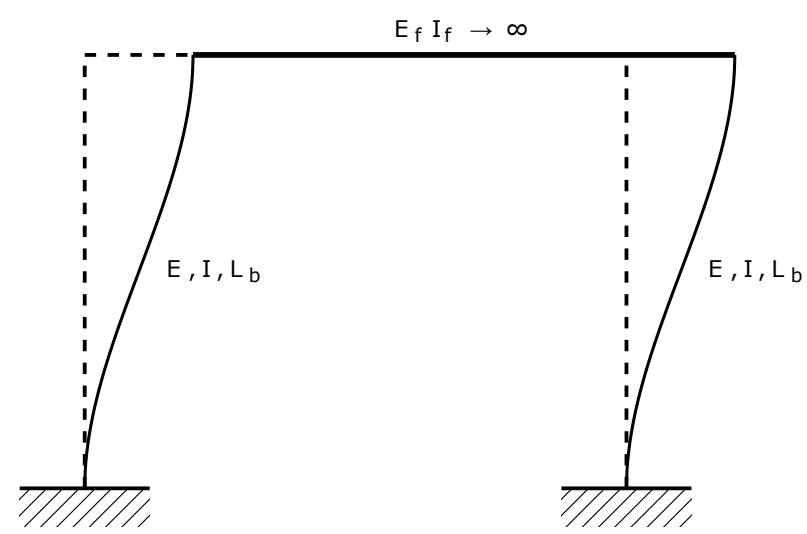

Figure 3: Deformation of the idealized model of the frame. The rigidity of the floor $\left(E_{f} I_{f}\right)$ is assumed to be infinity compared to connecting linear elastic elements.

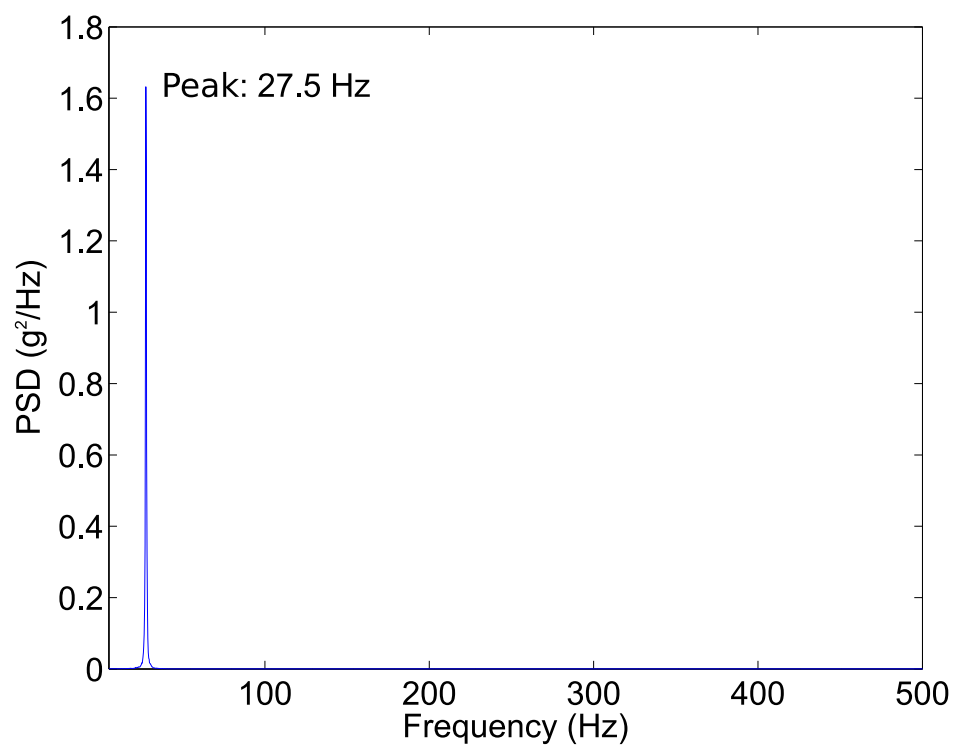

Figure 4: The power spectral density of the MS obtained from white noise.
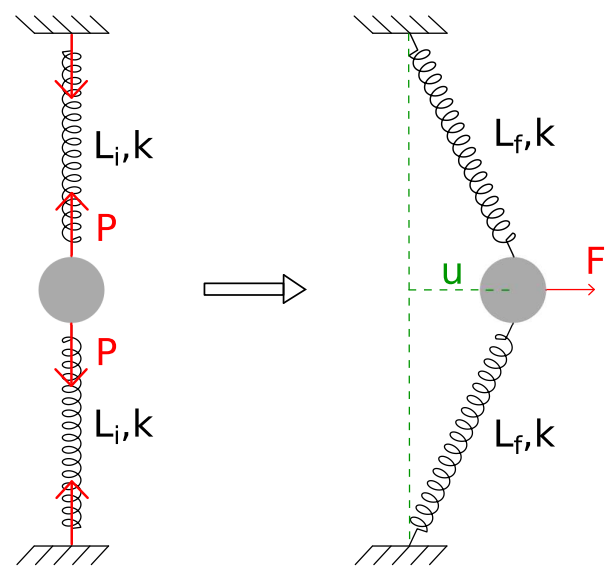

Figure 5: Realization of a cubic nonlinear restoring forcing function. 


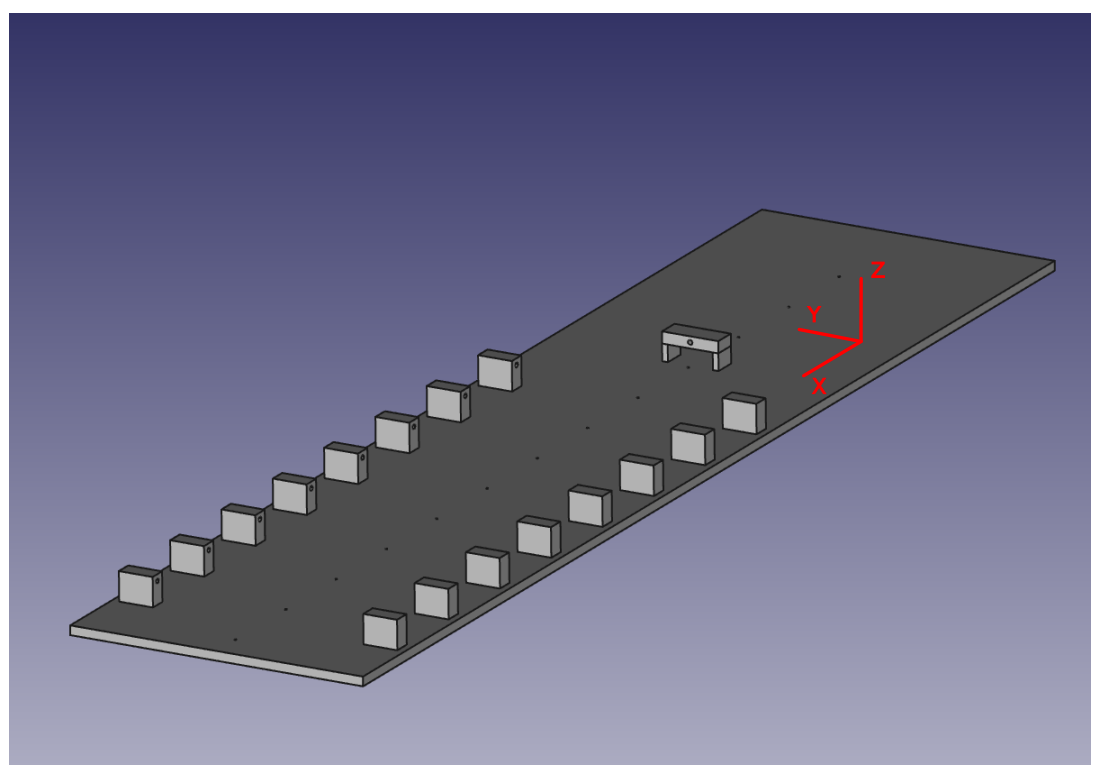

Figure 6: The schematic of the support plate of the nonlinear chain.

Equation 5 can be developed in the form of series versus $u$ as

$$
F=\frac{2 P}{L_{i}} u+\frac{k L_{i}-P}{L_{i}^{3}} u^{3}+\mathcal{O}\left(u^{5}\right)
$$

Considering that

$$
P=k\left(L_{i}-L_{0}\right)
$$

with $L_{0}$ being undeformed length of the the springs, then Eq. 7 can be reorganized as:

$$
F=2 k \frac{L_{i}-L_{0}}{L_{i}} u+\frac{k L_{0}}{L_{i}^{3}} u^{3}+\mathcal{O}\left(u^{5}\right)
$$

Equation 9 shows that the cubic restoring forcing function can be realized via coupling two linear springs to a mass moving in the transversal direction. It should be mentioned that if the system does not possess any pre-stressing terms i.e. $P=0$, then $L_{i}=L_{0}$; in this case Eq. 9 reads:

$$
F=\frac{k}{L_{i}^{2}} u^{3}+\mathcal{O}\left(u^{5}\right)
$$

Truncating $\mathcal{O}\left(u^{5}\right)$ in Eq. 10 provides an obvious pure cubic restoring forcing function with "no" linear part. The creation of the linear coupling between masses of the chain, i.e. global restoring forcing function $\tilde{B}(x-y)$ (see Eq. (1) with $x$ and $y$ standing for generalized displacements of two neighbor masses, can be carried out by using linear springs. Practically, springs without any pre-stressing act only on tension or on compression. Due to change of the sign of relative displacements of two neighbor masses, these linear springs can experience both tensions and compressions. In this case, the spring should be under pre-tension (or pre-compression) if it acts only in tension (or compression) in a way that each one should move until half of its maximum possible displacements, named as $S$. This will force that all springs act only in tension (or compression) provided that the relative displacements of the chain do not exceed $\frac{S}{2}$. For our experimental device we use the tensional springs for creation of linear coupling terms between: masses, the first mass of the chain and the main system, and the last mass of the chain and the end support.

For realization of the overall components of the chain, a plate which is shown in Fig. 6 is fabricated. Two rows of parallel connection supports are placed at both sides of the plate for coupling local springs of the chain. There is a single support at far end in the middle of the plate for connecting the last linear spring of the chain to the last mass. In the middle of the plate of Fig. 6 a rail is placed. A mobile cart is mounted on the rail for carrying masses of the chain. All masses of the chain are 3D-printed in resin (Printer: ProJet 3510SD of 3DSystems Europe Ltd., resin: VisiJet M3 Crystal), and are mounted on the mobile carts. The mass can be easily decomposed in order to place all connecting local and global springs. The overall view of all the system is presented in Fig 7 where chain of nonlinear oscillators is coupled to the main system via a linear spring. All connecting local and global springs to a single mass of the chain 


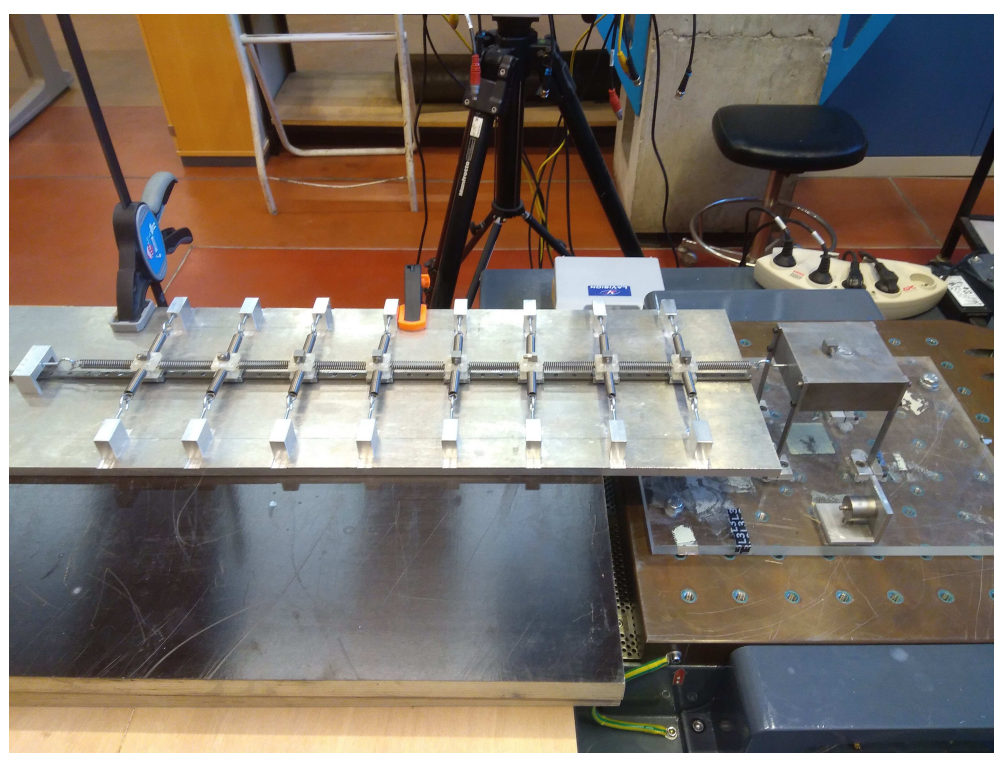

Figure 7: The overall system: the main oscillator is fixed on the shaking table while the supporting plate of the chain is placed on the static table.

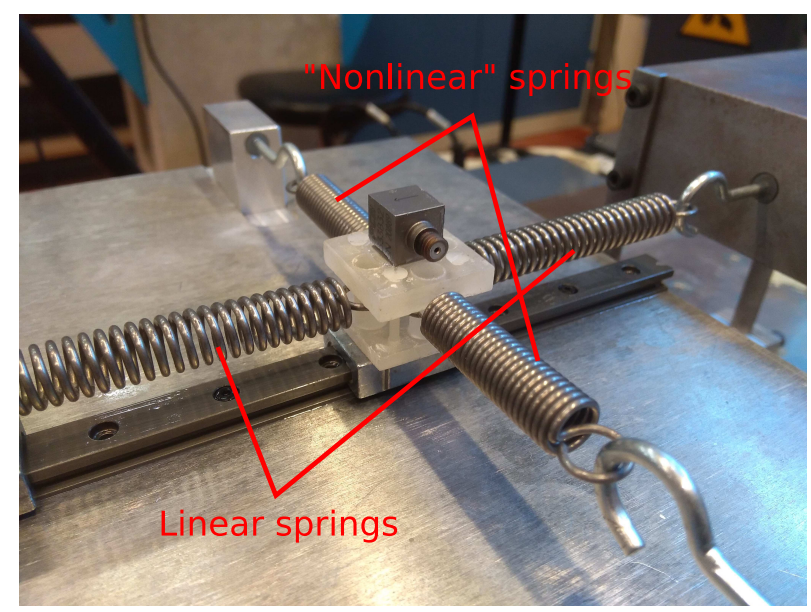

Figure 8: Local and global springs connecting to a mass which act as nonlinear and linear restoring forcing functions, respectively.

are shown in Fig. 8 As it is seen, the main system is mounted on the shaking table and the chain of the nonlinear oscillators is on top of a static table. The translation of the induced force of the main system to the chain is carried out by the linear coupling spring which connects the main system to the first mass of the chain.

\subsection{Characterization of the overall system}

For evaluating the total mass of each oscillators of the chain, we sum the masses of the mobile cart, printed mass on top of the cart and accelerometer of top of each printed mass. Then, the total mass of each oscillator reads $m=27.5$ g. The mass ratio copies $\epsilon=\frac{m}{M}=\frac{27.5 \times 10^{-3}}{3.18}=8.65 \times 10^{-3}$. The chain possesses eight oscillators in total where the overall mass of the chain is $m_{\text {overall }}=8 m=220 \mathrm{~g} \ll M$. The linear spring connecting oscillators of the chain has a coefficient as $\tilde{B}=2420 \mathrm{~N} \cdot \mathrm{m}^{-1}$ and its length at rest position is $L_{0}=51 \mathrm{~mm}$. These springs are also endowed for creation of local nonlinear springs. Taking into account the effects of the pre-tensioning of springs with $L_{1}-L_{0}=1$ 
$\mathrm{mm}$ and considering Eq. 9 we have:

$$
F(u)=2 \times 2420 \times \frac{1 \times 10^{-3}}{52 \times 10^{-3}} u+2420 \times \frac{51 \times 10^{-3}}{\left(52 \times 10^{-3}\right)^{3}} u^{3}+\mathcal{O}\left(u^{5}\right)
$$

or after truncating $\mathcal{O}\left(u^{5}\right)$ :

$$
F(u)=\tilde{B}_{l} u+\tilde{V} u^{3}=93.1 u+8.78 \times 10^{5} u^{3}
$$

Other system parameters are summarized in Table 2 . Since the aim of the current work is to analyse behaviour of the

\begin{tabular}{|c|c|c|c|c|c|}
\hline$c$ & $\gamma$ & $f$ & $B$ & $B_{l}$ & $D$ \\
\hline $0.23 C$ & $0.23 \Gamma$ & $0.15 A_{M S}$ & 3.57 & 0.14 & 0.13 \\
\hline
\end{tabular}

Table 2: Characteristics of the overall system

overall system experimentally, we do not need to identify constants $C$ and $\Gamma$.

\section{Experimental tests on the chain and on the coupled systems}

Globally two types of systems are experimented: i) the first global spring of the chain in connected directly to the shaking table; ii) the main system is mounted on the shaking table and the chain is coupled to the main system via the first global linear spring. The idea is to obtain (qualitatively) explanations of the behaviours of the chain alone and then to relate these behaviours on the responses of the coupled systems. In the next section results of each case are presented and commented upon.

\subsection{Experimental tests on the chain}

The first linear spring of the chain is connected to the shaking table directly. Different sinusoidal excitations with constant frequency as $\nu=\frac{\omega}{2 \pi}=27.5 \mathrm{~Hz}$ representing the frequency of the first mode of the main system, see Fig. 4 . and constant amplitudes $A_{f}$, simulating the amplitude of the vibration of the main system, are applied. Several tests are carried out applying different forcing amplitudes $A_{f} \in[5,40] \mathrm{m} \cdot \mathrm{s}^{-2}$. At each test, the maximum acceleration of each mass named as $A_{j}, j=1,2, \ldots, 8$, are collected which are related to periodic behaviour of each mass during the external excitation with the amplitude $A_{f}$. Maximum acceleration of first three masses of the chain, i.e. $A_{1}, A_{2}$ and $A_{3}$, during the periodic behaviours are presented in Figs. 9-11 Each point is collected from one experimental test corresponding to one acceleration of the external excitation $A_{f}$ and of the mass $A_{j}$, representing amplitudes of the forcing term and masses, respectively. Figures 9 11 gather all periodic behaviours of the system for different external forcing terms which in fact correspond to experimentally obtained slow invariant manifold (SIM) (12) of the system, denoted ESIM. These figures identify two distinguished thresholds of external forcing amplitudes: the first one corresponds to $A_{f} \in[17.5,18] \mathrm{m} . \mathrm{s}^{-2}$ and the second one belongs to $A_{f} \in[34,35] \mathrm{m} . \mathrm{s}^{-2}$. We name them as $(\mathfrak{t})_{1}$ and $(\mathbb{t})_{2}$, respectively. At $\left(\mathfrak{t}_{1}\right.$ the first mass experiences a bifurcation with abrupt and large variations for $A_{1}$ and smaller variations for $A_{2}$ and $A_{3}$. This corresponds to variations between two stable branches of the ESIM, named as first and second branches, respectively (12). The $\mathfrak{t}_{2}$ corresponds to another bifurcation of the system representing large variations for $A_{2}$ and $A_{3}$ and the moderate one for $A_{1}$. This is performed between second and third branches of the ESIM (12). The maximum acceleration of each mass of the nonlinear chain corresponding to external excitation in $\left(\mathfrak{t}_{1}\right.$ and $\mathfrak{t}_{2}$ are provided in Figs. 12 and 13 , respectively. It is seen that first three masses of the chain present evident bifurcations between extremes of thresholds $\mathfrak{t}_{1}$ and $\mathfrak{t}_{2}$ while bifurcations of other masses are not evident. This is probably due to the nature of the ESIM of the system which would present different amplitude variations for each mass of the chain (12).

\subsection{Experimental tests on the complete system: the main oscillator coupled to the nonlinear chain}

The MS is mounted on the shaking table and the chain is coupled to the MS via the linear global spring, see Fig. 7 The MS is subjected to sinusoidal excitation with the sweeping frequency as $\nu \in[27,28.5] \mathrm{Hz}$ and with different amplitudes as $A_{f} \in\{0.1,0.2,0.3,0.4,0.5,0.6\} \mathrm{m}_{\mathrm{s}}{ }^{-2}$. Collected experimental amplitudes of the MS (in m.s $\mathrm{s}^{-2}$ ), for without and with coupled chain, and also amplitudes of the first three masses of the chain, $A_{j}, j=1,2,3$, are illustrated in Fig. 14 When the amplitude of the excitation is low, i.e. $A_{f}=0.1 \mathrm{~m} . \mathrm{s}^{-2}$, we observe that: i) the overall system, i.e. MS and the coupled mass, present a linear resonant frequency which is higher than the one of the MS alone, see Fig. $14 \mathrm{a}$ and ii) the amplitude of MS at peak presents $11.5 \%$ of augmentation, while the $A_{j}, j=1,2 ; \ldots, 8$ 


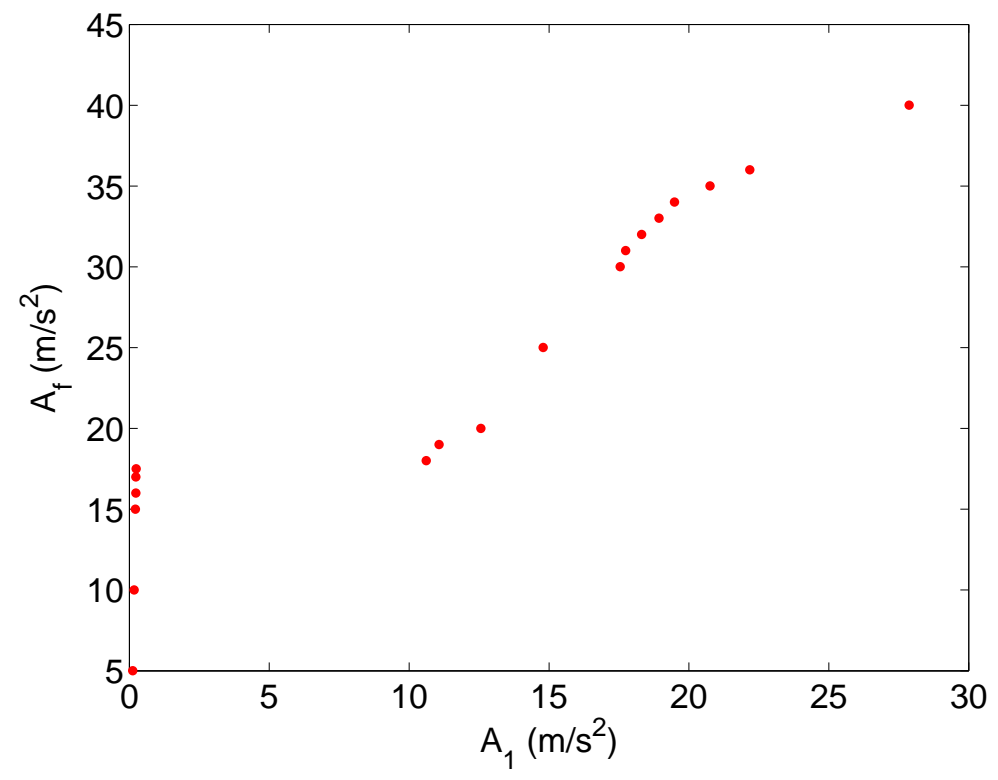

Figure 9: Results of experimental sinusoidal test on the nonlinear chain: The maximum amplitude of the first mass, $A_{1}$ during its periodic behaviours versus the amplitude of the excitation $A_{f}$. The test is carried out with different forcing amplitudes $A_{f}$ and constant frequency $\nu=27.5 \mathrm{~Hz}$. Each point corresponds to the result of one experimental test.

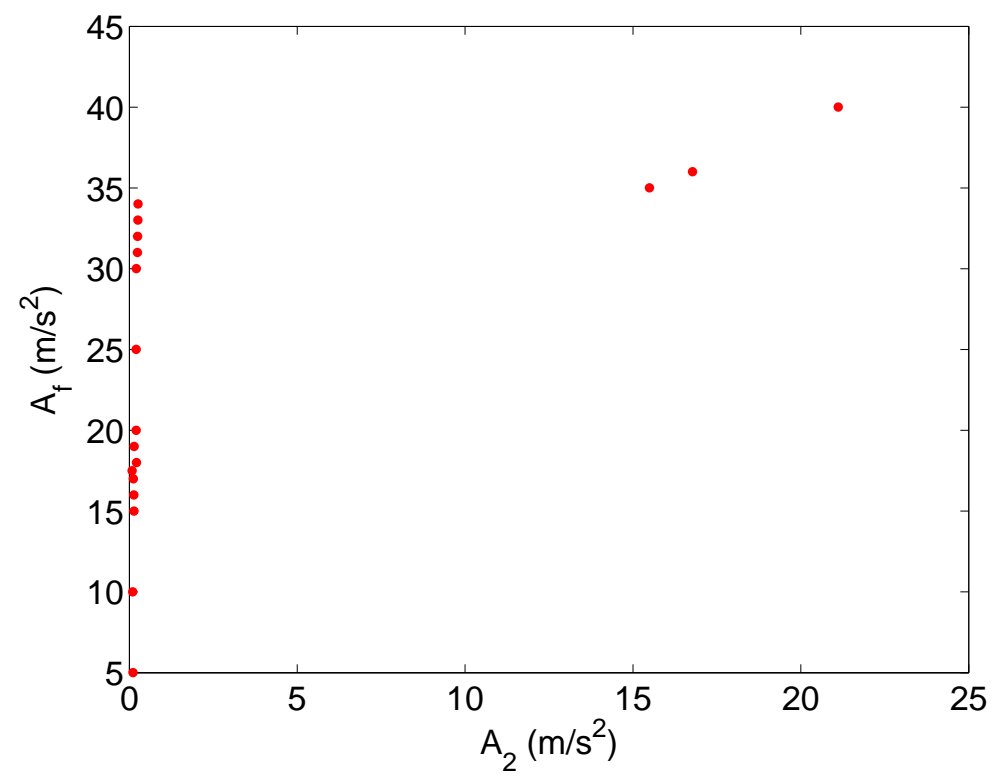

Figure 10: Results of experimental sinusoidal test on the nonlinear chain: The maximum amplitude of the second mass, $A_{2}$ during its periodic behaviours versus the amplitude of the excitation $A_{f}$. The test is carried out with different forcing amplitudes $A_{f}$ and constant frequency $\nu=27.5 \mathrm{~Hz}$. Each point corresponds to the result of one experimental test. 


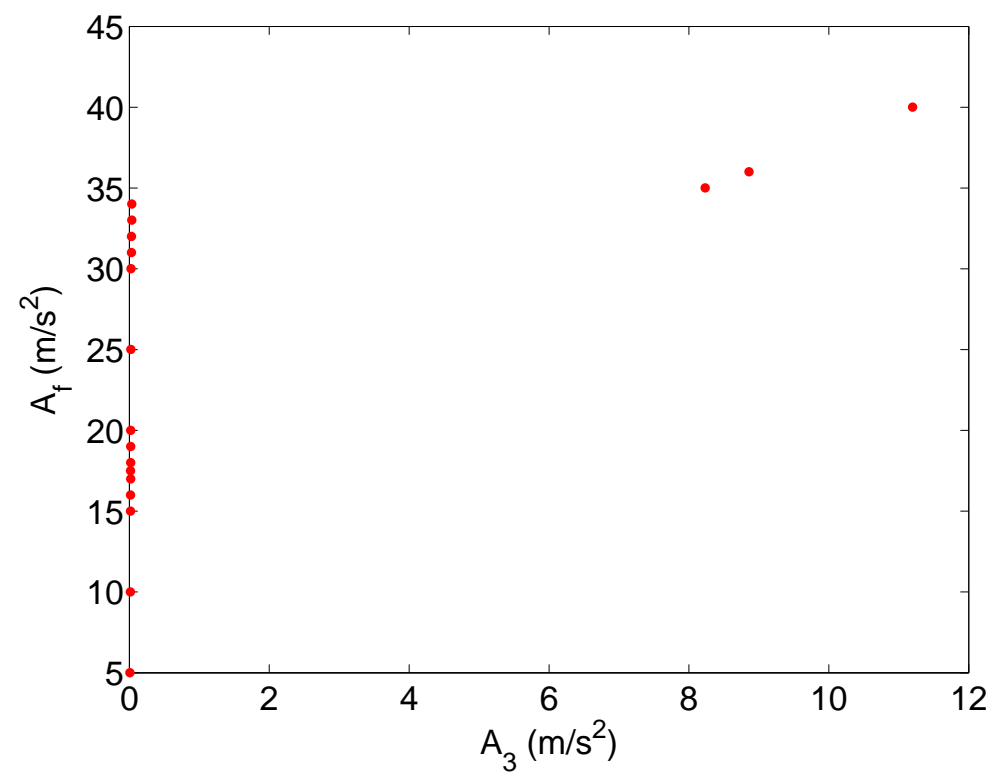

Figure 11: Results of experimental sinusoidal test on the nonlinear chain: The maximum amplitude of the third mass, $A_{3}$ during its periodic behaviours versus the amplitude of the excitation $A_{f}$. The test is carried out with different forcing amplitudes $A_{f}$ and constant frequency $\nu=27.5 \mathrm{~Hz}$. Each point corresponds to the result of one experimental test.

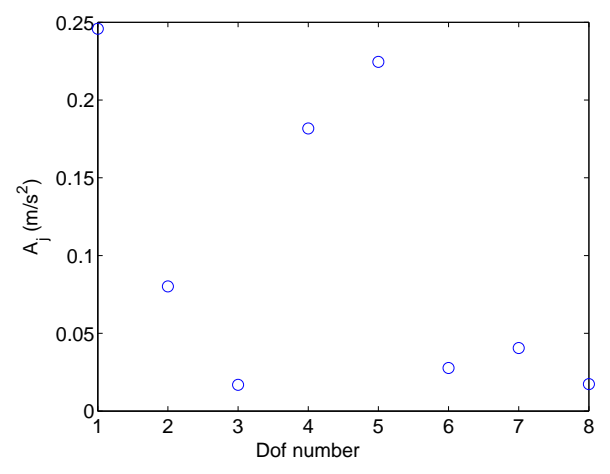

(a) $A_{f}=17.5 \mathrm{~m} \cdot \mathrm{s}^{-2}$

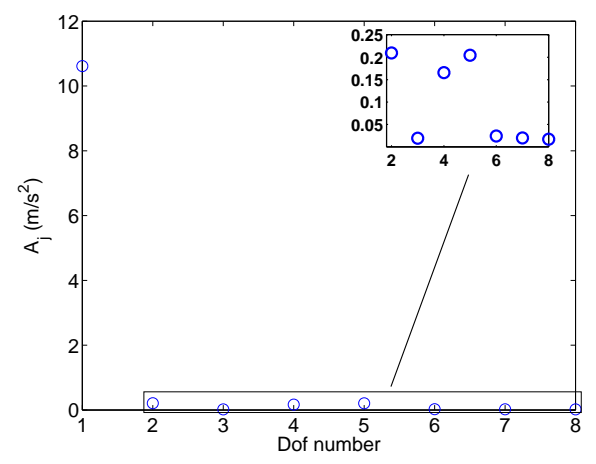

(b) $A_{f}=18 m . s^{-2}$

Figure 12: Maximum accelerations of each mass of the chain (each dof) for threshold $\mathbb{t}_{1}$ of the external forcing amplitude. 


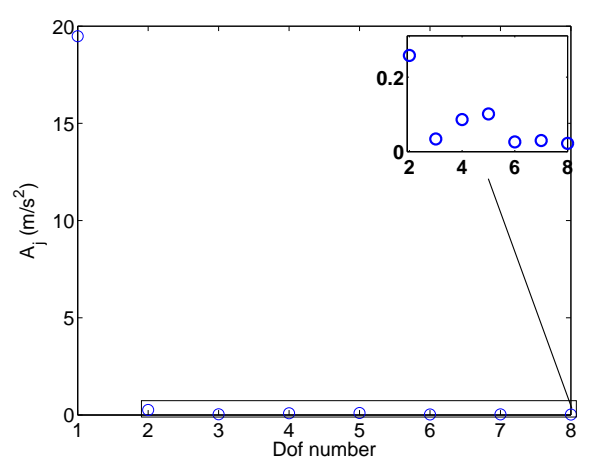

(a) $A_{f}=34 m . s^{-2}$

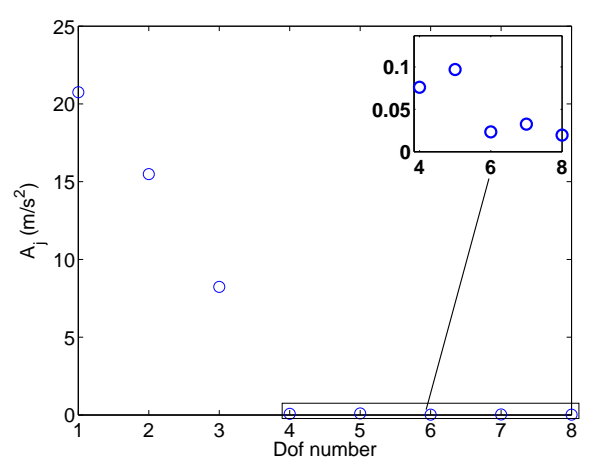

(b) $A_{f}=35 m \cdot s^{-2}$

Figure 13: Maximum accelerations of each mass of the chain (each dof) for threshold $\mathbb{t}_{2}$ of the external forcing amplitude.

stay almost at the same levels of amplitudes of before the threshold $\mathbb{t}_{1}$ which has been clarified in Sect. 4.1. Figure $14 \mathrm{~b}$ presents frequency response of the $A_{M S}$ for $A_{f}=0.2 \mathrm{~m} . \mathrm{s}^{-2}$. The system presents the same type of behaviour as explained for the first case, but at $\nu=27.98 \mathrm{~Hz}$, there is an abrupt reduction on the $A_{M S}$ which houses a jump for $A_{1}$. This bifurcation is carried out between the first and the second branches of the ESIM and corresponds to the start of the threshold $\mathbb{t}_{1}$ which is more evident for $A_{1}$ (see Figs. 9, $[12$ ). The behaviour of the system changes radically when the applied external forcing amplitude reads as $A_{f}=0.3 \mathrm{~m} . \mathrm{s}^{-2}$. As depicted in Fig. 14c during the frequency interval $\nu \in[27.9,28.12] \mathrm{Hz}$, the system shows repeated amplitude variations between (around) $A_{M S}=19$ and $27 \mathrm{~m} . \mathrm{s}^{-2}$. Time histories of the $A_{m s}$ and $A_{j}, j=1,2,4$ for the system with excitation of the table with the amplitude of $A_{f}=0.3$ m.s $\mathrm{s}^{-2}$ and frequency as $\nu=28 \mathrm{~Hz}$ are provided in Fig. 15. This behaviour is a Strongly Modulated Response (17) corresponding to repeated bifurcations between the first and the second branches of the ESIM characterized by the threshold $\left(\mathrm{C}_{1}\right.$. The system responses for the amplitude of excitation of $A_{f}=0.4 \mathrm{~m} . \mathrm{s}^{-2}$ which is given in Fig. $14 \mathrm{~d}$ shows that the system bifurcates to the second branch of the ESIM corresponding to the threshold $(\mathbb{t})_{1}$ at the frequency of $\nu=27.8 \mathrm{~Hz}$ which is accompanied by an abrupt reduction of the $A_{M S}$ and sudden increase of the $A_{1}$. Then, by increasing the frequency the system follows the second branch of the ESIM which corresponds to periodic regimes and also the reduction of the energy for the main system. The system bifurcates at $\nu=28.28 \mathrm{~Hz}$ to the first branch of the ESIM and presents again periodic responses. The response of the system under amplitude excitation of $A_{f}=0.5$ m.s $\mathrm{s}^{-2}$ is presented in Fig. 14e At the frequency $\nu=27.78 \mathrm{~Hz}$ the system bifurcates to the second branch of the ESIM and at $\nu=27.8 \mathrm{~Hz}$ it jumps to the third branch of the ESIM, corresponding to $\mathbb{C}_{2}$, which is accompanied by large movements of the second mass of the chain. The MS increases its energy on the third branch during periodic regimes until $\nu=27.93 \mathrm{~Hz}$, then it follows the third branch of the ESIM during periodic regimes in the reverse direction via lowering the energy of the MS until $\nu=28.06 \mathrm{~Hz}$. Here, the system jumps to the second branch of the ESIM and lowers the energy of the MS until $\nu=28.26 \mathrm{~Hz}$. At this frequency, the system bifurcates to the first branch of the ESIM housing the increase of the $A_{M S}$ and decrease of the $A_{1}$ and $A_{2}$. Afterwards, $A_{M S}$ decreases during periodic regimes. The system under excitation with $A_{f}=0.6 \mathrm{~m} . \mathrm{s}^{-2}$ presents the same type of behaviour which is explained in previous case but here the variations of the energy of the third oscillator of the chain $A_{3}$ are large, see Fig. 14f

\section{Conclusions}

Experimental results are carried out on a system which is composed of a main linear oscillator, representing a targeted mode to be controlled, coupled to a nonlinear chain of oscillators. The chain possesses eight mobile light masses under local nonlinear and global linear restoring forces. The main system has been mounted on the shaking table and is excited with several amplitudes and a sweeping frequency around its targeted mode, i.e. the one to be controlled. Two distinct thresholds are spotted corresponding to different branches of the slow invariant manifold (12) of the system. The presented results shows that according to the amplitude of the excitation the system can present periodic regimes (18) or strongly modulated responses (17). For having refined design tools, a detailed analytical developments should be carried out to trace the analytical slow invariant manifold and equilibrium and singular points of the system which will be future developments of the current work. Using a multiple degrees of freedom nonlinear oscillators instead of the single one can have practical reasons such as: distributing the mass and the rigidities of a single oscillator into several ones and also increasing robustness of the system in the case that one (or even some) of the oscillators does not operate well or loses its (their) efficiency. 


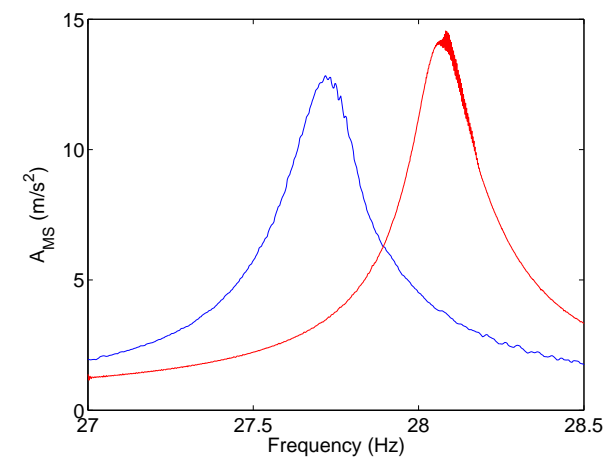

(a) $A_{f}=0.1 \mathrm{~m} \cdot \mathrm{s}^{-2}$

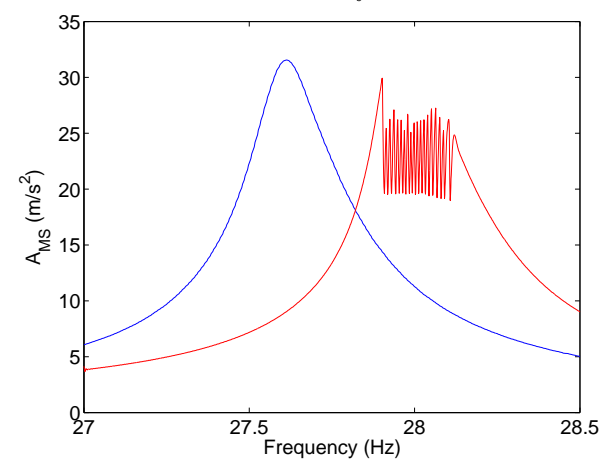

(c) $A_{f}=0.3 \mathrm{~m} \cdot \mathrm{s}^{-2}$

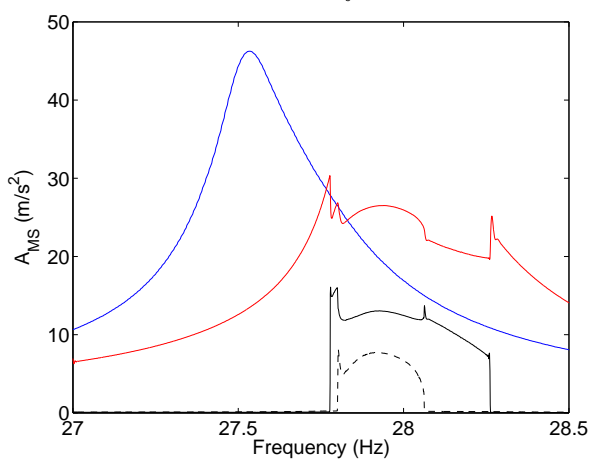

(e) $A_{f}=0.5 \mathrm{~m} \cdot \mathrm{s}^{-2}$

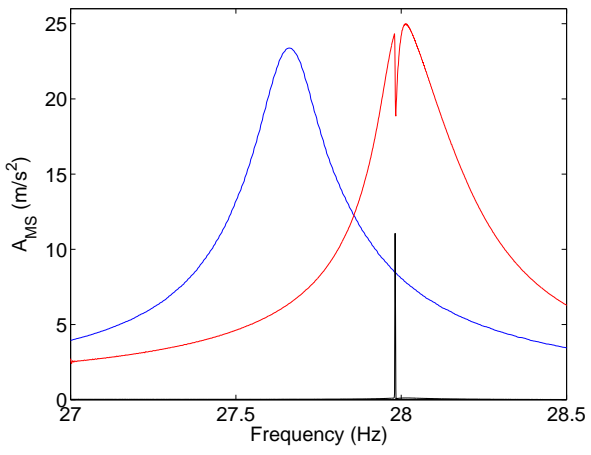

(b) $A_{f}=0.2 m . s^{-2}$

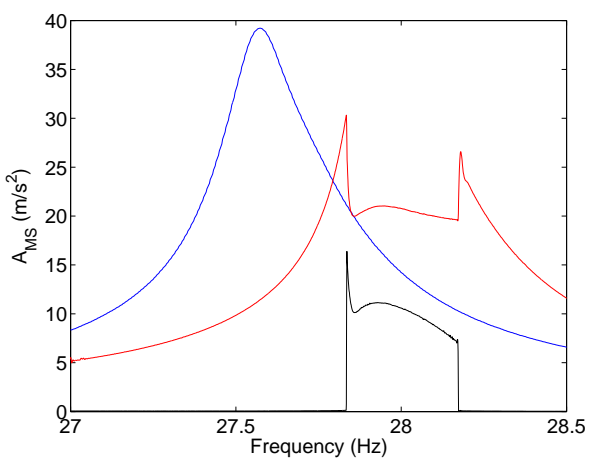

(d) $A_{f}=0.4 m . s^{-2}$

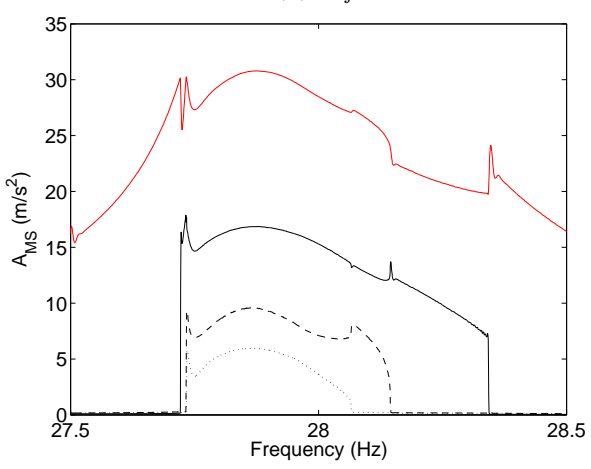

(f) $A_{f}=0.6 m \cdot s^{-2}$

Figure 14: Experimental results obtained from sinusoidal tests with sweeping frequency in $\nu \in[27,28.5] \mathrm{Hz}$ and excitation amplitudes $A_{f} \in\{0.1,0.2,0.3,0.4,0.5,0.6\} \mathrm{m} . \mathrm{s}^{-2}$. Blue and red lines stand for the maximum amplitude responses of the MS $\left(A_{M S}\right)$, without and with coupled nonlinear chain. The responses of the first, second and third masses of the chain are represented by black solid, dashed and dotted lines, respectively. 


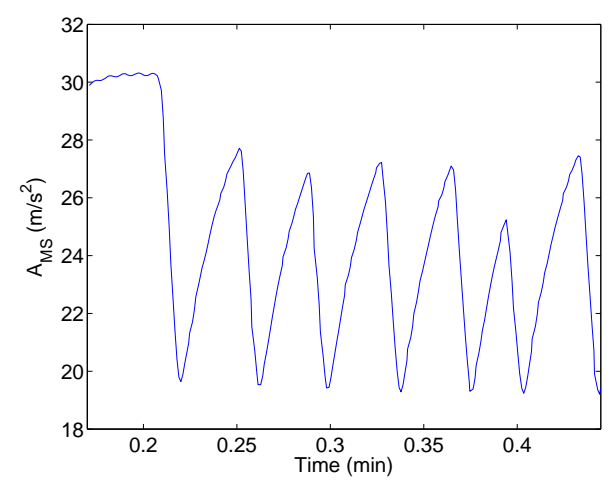

(a) $A_{M S}$

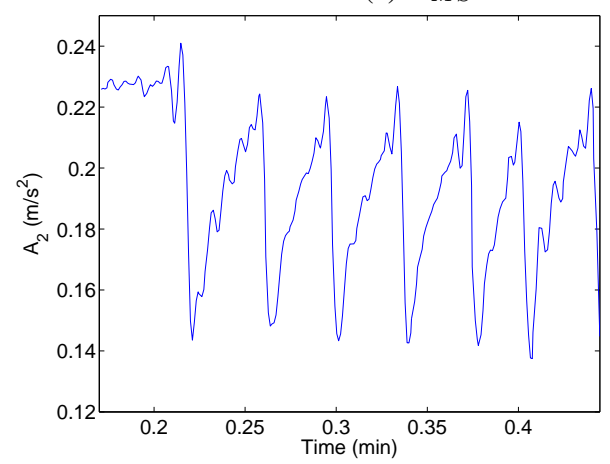

(c) $A_{2}$

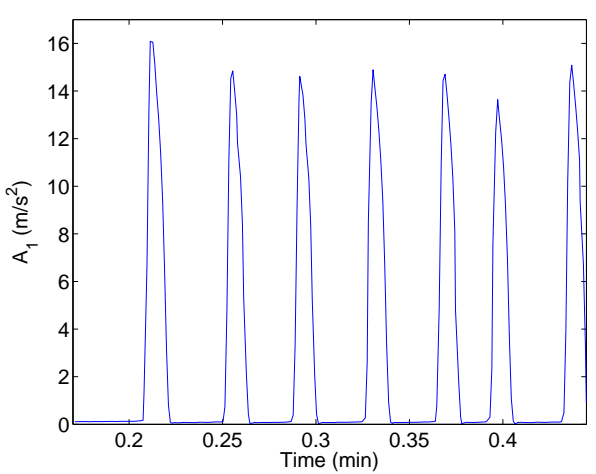

(b) $A_{1}$

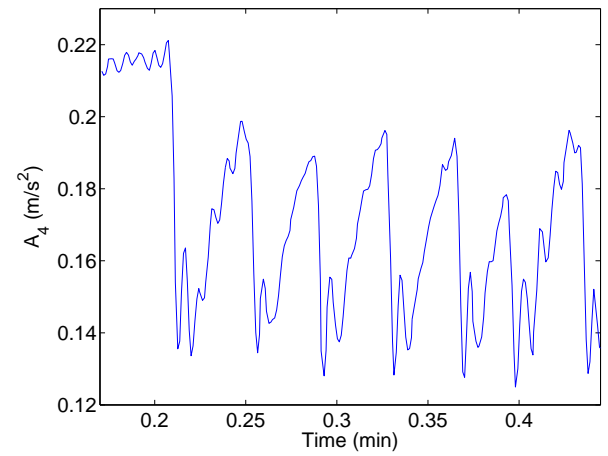

(d) $A_{4}$

Figure 15: Time histories of the maximum values of the amplitudes of the $A_{M S}$ and $A_{j}, j=1,2,4$ for the system under sinusoidal excitation with $A_{f}=0.3$ and the frequency $\nu=28 \mathrm{~Hz}$.

\section{Acknowledgments}

The authors would like to thank following organizations for supporting this research: i) The "Ministère de la Transition Écologique et Solidaire" ii) LABEX CELYA (ANR-10-LABX-0060) of the "Université de Lyon" within the program "Investissement d'Avenir" (ANR-11-IDEX-0007) operated by the French National Research Agency (ANR).

\section{Appendix}

\section{A Steps for analytical treatments of Eq. 2}

The main goal is to trace characteristics points of the system leading to periodic and modulated regimes. To this end, following steps can be applied for treatments of the system 2, $(10,12)$ :

- a change of variables can be applied. For instance, relative generalized displacements can be introduced:

$$
\left\{\begin{array}{l}
w_{1}=\tilde{u}_{1}-\tilde{v} \\
w_{j}=\tilde{u}_{j}-\tilde{u}_{j-1}, \quad j=2, \ldots, L+1
\end{array}\right.
$$

- complex variables of Manevitch (20) are injected to system variables:

$$
\boldsymbol{\Phi} e^{i \omega t}=\dot{\mathbf{y}}+i \omega \mathbf{y}
$$

where

$$
\mathbf{y}^{T}=\left(\begin{array}{lllll}
\tilde{v} & w_{1} & w_{2} & \ldots & w_{L+1}
\end{array}\right)
$$

- A time multiple-scale method (21) is endowed to treat the system responses at different scales of time. In detail: the tme $t$ is embedded to different scales namely fast connecting to each other via the $\epsilon$ parameter. They read as fast $\tau_{0}=T$ and slow scales $\tau_{k}=\epsilon^{k} T, k=1,2, \ldots$ 
- The truncated Fourier series is retained by keeping only first harmonics of the system. For an arbitrary function $\mathcal{R}$, this task is achieved via

$$
\Upsilon=\frac{\omega}{2 \pi} \int_{0}^{\frac{2 \pi}{\omega}} \mathcal{R}\left(\tau_{0}, \tau_{1}, \tau_{2}, \ldots\right) e^{-i \omega \tau_{0}} \mathrm{~d} \tau_{0}
$$

During the application of Eq. 16 it is assumed that the $\boldsymbol{\Phi}$ is independent of the fast time scale $\tau_{0}$. This is verified during the multiple scale method or when we seek the behaviours of the system during its fixed points where $\frac{\mathrm{d} \Phi}{\mathrm{d} \tau_{0}}=0$.

- We consider the case of the 1:1 resonance; we set $\Omega=1+\epsilon \sigma$.

- The $\epsilon^{0}$ order of system equations, i.e. fast equations, provide the slow invariant manifold (SIM) of the system. It is the geometrical invariant for all types of system behaviours when $\tau_{0} \rightarrow \infty$.

- The $\epsilon^{1}$ order of system equations, i.e. slow equations, around the SIM provide equilibrium and singular points. They correspond to periodic and modulated (17) regimes of the system.

\section{References}

[1] R. E. Roberson, 1952. Synthesis of a nonlinear dynamic vibration absorber, Journal of The Franklin Institute 254, 205-220.

[2] O. V. Gendelman, 2011. Targeted energy transfer in systems with external and self excitation, Proceedings of the Institution of Mechanical Engineers, Part C: Journal of Mechanical Engineering Science 225 (9), 2007-2043.

[3] A. Luongo, D. Zulli, 2014. Aeroelastic instability analysis of NES-controlled systems via a mixed multiple scale/harmonic balance method, Journal of Vibration and Control 20, 1985-1998.

[4] O. V. Gendelman, A. Alloni, 2014. Dynamics of forced system with vibro-impact energy sink, Journal of Sound and Vibration 358, 301-314.

[5] P. N. Panagopoulos, A. F. Vakakis, S. Tsakirtzis, 2004. Transient resonant interactions of finite linear chains with essentially nonlinear end attachments leading to passive energy pumping, International Journal of Solids and Structures 41 (2223), 6505-6528.

[6] S. Tsakirtzis, G. Kerschen, P. N. Panagopoulos, A. F. Vakakis, 2005. Multifrequency nonlinear energy transfer from linear oscillators to mdof essentially nonlinear attachments, Journal of Sound and Vibration 285 (12), 483490.

[7] S. Tsakirtzis, P. N. Panagopoulos, G. Kerschen, O. Gendelman, A. F. Vakakis, L. A. Bergman, 2007. Complex dynamics and targeted energy transfer in linear oscillators coupled to multi-degree-of-freedom essentially nonlinear attachments, Nonlinear Dynamics 48 (3), 285-318.

[8] L. I. Manevitch, A. Kovaleva, V. Smirnov, Y. Starosvetsky, 2018. Nonstationary Resonant Dynamics of Oscillatory Chains and Nanostructures, Springer, 436 pages.

[9] Y. Starosvetsky, K. R. Jayaprakash, M. A. Hasan, A. F. Vakakis, 2017. Topics on the Nonlinear Dynamics and Acoustics of Ordered Granular Media, World Scientific Publishing Company, 639 pages.

[10] S. Charlemagne, C.-H. Lamarque, A. Ture Savadkoohi, 2017. Vibratory control of a linear system by addition of a chain of nonlinear oscillators, Acta Mechanica 228, 3111-3133.

[11] C.-H. Lamarque, A. Ture Savadkoohi, S. Charlemagne, 2017. Passive control of a linear structure via nonlinear oscillators in series, 14th International Conference: Dynamical Systems-Theory and Applications, pp. 269-278, Lodz, Poland.

[12] S. Charlemagne, A. Ture Savadkoohi, C.-H. Lamarque, 2018. Dynamics of a linear system coupled to a chain of light nonlinear oscillators analyzed through a continuous approximation, Physica D 374-375, 10-20.

[13] O. V. Gendelman, Y. Starosvetsky, M. Feldman, 2008. Attractors of harmonically forced linear oscillator with attached nonlinear energy sink I: Description of response regimes, Nonlinear Dynamics 51, pp. 31-46.

[14] D. M McFarland, L. A. Bergman, A. F. Vakakis, 2005. Experimental study of non-linear energy pumping occurring at a single fast frequency, International Journal of Non-Linear Mechanics 40 (6), 891-899.

[15] E. Gourdon, N. A. Alexander, C. A. Taylor, C.-H. Lamarque, S. Pernot, 2007. Nonlinear energy pumping under transient forcing with strongly nonlinear coupling: Theoretical and experimental results, Journal of Sound and Vibration 300, 522-551. 
[16] A. Ture Savadkoohi, B. Vaurigaud, C.-H. Lamarque, S. Pernot, 2012. Targeted energy transfer with parallel nonlinear energy sinks, part II: theory and experiments, Nonlinear Dynamics 67 (1), 37-46.

[17] Y. Starosvetsky, O.V. Gendelman, 2008. Strongly modulated response in forced 2DOF oscillatory system with essential mass and potential asymmetry, Physica D 237 (13), 1719-17339.

[18] A. Ture Savadkoohi, C.-H. Lamarque, M. Weiss, B. Vaurigaud, S. Charlemagne, 2016. Analysis of the 1:1 resonant energy exchanges between coupled oscillators with rheologies, Nonlinear Dynamics 86 (4), 3111-3133.

[19] A. K. Chopra, 2012. Dynamics of Structures: Theory and Applications to Earthquake Engineering, Prentice Hall, 944 pages.

[20] L. I. Manevitch, 2001. The description of localized normal modes in a chain of nonlinear coupled oscillators using complex variables, Nonlinear Dynamics 25 (1-3), 95-109.

[21] A.H. Nayfeh, D.T. Mook, 1979. Nonlinear oscillations, John Wiley and Sons, 704 pages. 